\title{
Interactive comment on "Effect of Atmospheric Stability on the Wind Resource extrapolating models for large capacity Wind Turbines: A Comparative Analysis of Power Law, Log Law and Deaves and Harris mode" by Pramod Kumar Sharma et al.
}

\section{Anonymous Referee \#2}

\section{Received and published: 17 May 2018}

While the area of study is certainly relevant, I agree with Reviewer 1 that it is quite difficult to assess the scientific merits of this work due to the poor quality of writing.

I strongly suggest that the authors seek proofreading services and resubmit. The abstract of the revised version posted here still contains many grammatical errors. 